\title{
Office-Based Blue Light Flexible Cystoscopy Improves Diagnostic Capabilities
}

\author{
Xiaosong Meng, MD, PhD \\ Department of Urology, UT Southwestern Medical Center, Dallas, Texas. \\ Iftach Chaplin, MD \\ Department of Urology, UT Southwestern Medical Center, Dallas, Texas. \\ Rashed Ghandour, MD \\ Department of Urology, UT Southwestern Medical Center, Dallas, Texas. \\ Yair Lotan, MD \\ Department of Urology, UT Southwestern Medical Center, Dallas, Texas. \\ E-mail:Yair.lotan@utsouthwestern.edu
}

() Mary Ann Liebert, Inc. DOI: 10.1089/vid.2019.0081
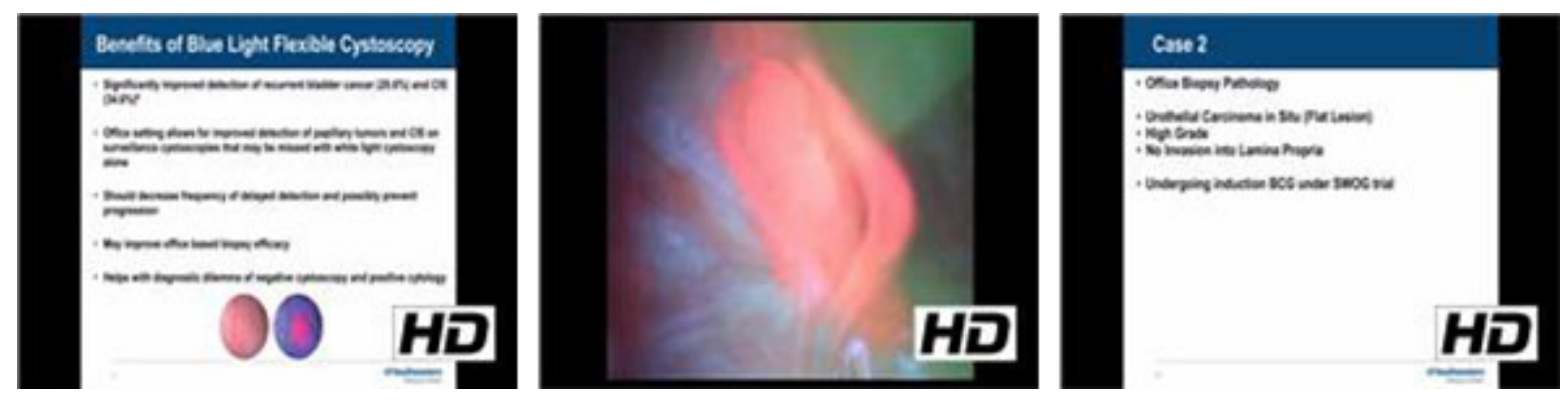

\begin{abstract}
Introduction: Bladder cancer is associated with a $78 \%$ risk of recurrence at 5 years for nonmuscleinvasive bladder cancer and $45 \%$ progression to muscle-invasive disease. ${ }^{1}$ This is common in patients with carcinoma in situ (CIS), which can often be missed with standard white light cystoscopy. ${ }^{2}$ Additionally, $34-76 \%$ of patients have evidence of residual tumors after transurethral resection of bladder tumor ${ }^{3,5}$ and patients with incomplete initial resection are at a high risk of recurrence ${ }^{4,6,7}$ Blue light flexible cystoscopy in the office setting improves detection of papillary tumors and $\mathrm{CIS}^{8}{ }^{8}$ potentially decreasing frequency of delayed detection, possibly prevents disease progression and may improve office-based biopsy efficacy. In this study we present a video demonstrating how blue light flexible cystoscopy can be used in the office setting to assist with detection of papillary tumors and CIS.
\end{abstract}

Materials and Methods: Patients received hexaminolevulinate intravesically before white light flexible cystoscopy and blue light flexible cystoscopy. Suspicious lesions were biopsied with flexible graspers and fulgurated in the office setting when feasible.

Results: Patient 1 had a history of high-grade T1 bladder cancer treated with multiple prior surgeries and induction/maintenance Bacillus Calmette Guerin (BCG) with multiple flat lesions that were difficult to observe on white light cystoscopy but readily apparent under blue light. Biopsy demonstrated highgrade urothelial carcinoma. Patient 2 was incidentally found to have focus of CIS involving the bladder neck/prostatic urethra on bipolar transurethral resection of the prostate. Blue light cystoscopy identified a patch lateral to the left ureteral orifice, which demonstrated CIS on office biopsy. Patient 3 had a history of CIS treated with induction and maintenance BCG without recurrence since 2016. Blue light cystoscopy identified small papillary lesions, including one not seen on white light cystoscopy that were biopsied and fulgurated with pathology analysis demonstrating low-grade Ta bladder cancer. 
Conclusion: Blue light flexible cystoscopy with biopsy/fulguration can significantly improve detection in the office setting. This helps establish the diagnosis and decreases costs and morbidity by potentially avoiding trips to the operating room for patients with CIS or low-grade tumors.

Patient Consent Statement: All blue light flexible cystoscope procedures were performed after approval by the institutional review board, and after obtaining informed consent for the procedure from the patient. Author(s) have received and archived patient consent for video recording/publication in advance of video recording of procedure.

X.M., I.C., and R.G. have no conflicts of interest. Y.L. reports personal fees from Photocure, during the conduct of the study; grants from Storz, outside the submitted work.

Runtime of video: 6 mins 39 secs

Keywords: bladder neoplasm, flexible cystoscopy, blue light

\section{Cite this video}

Xiaosong Meng, Iftach Chaplin, Rashed Ghandour, Yair Lotan, Office-Based Blue Light Flexible Cystoscopy Improves Diagnostic Capabilities, Videourology. 2020, DOI: 10.1089/vid.2019.0081.

\section{References}

1. Sylvester RJ, van der Meijden APM, Oosterlinck W, et al. Predicting recurrence and progression in individual patients with stage Ta T1 bladder cancer using EORTC risk tables: A combined analysis of 2596 patients from seven EORTC trials. Eur Urol 2006;49:466-477.

2. Babjuk $M$, Oosterlinck $W$, Sylvester $R$, et al. EAU guidelines on non-muscle-invasive urothelial carcinoma of the bladder, the 2011 update. Eur Urol 2011;59:997-1008.

3. Herr HW. The value of a second transurethral resection in evaluating patients with bladder tumors. J Urol 1999;162:74-76.

4. Divrik RT, Yildirim Ü, Zorlu F, Özen $H$. The effect of repeat transurethral resection on recurrence and progression rates in patients with $\mathrm{t} 1$ tumors of the bladder who received intravesical mitomycin: A prospective, randomized clinical trial. J Urol 2006;175:1641-1644.

5. Adiyat KT, Katkoori D, Soloway CT, De Los Santos R, Manoharan M, Soloway MS. "Complete transurethral resection of bladder tumor": Are the guidelines being followed? Urology 2010;75:365367.

6. Jocham D, Witjes F, Wagner S, et al. Improved detection and treatment of bladder cancer using hexaminolevulinate imaging: a prospective, phase III multicenter study. J Urol 2005;174:862-866.

7. Brausi $\mathrm{M}$, Collette L, Kurth $\mathrm{K}$, et al. Variability in the recurrence rate at first follow-up cystoscopy after TUR in stage Ta T1 transitional cell carcinoma of the bladder: a combined analysis of seven EORTC studies. Eur Urol 2002;41:523-531.

8. Daneshmand S, Patel S, Lotan Y, et al. Efficacy and safety of blue light flexible cystoscopy with hexaminolevulinate in the surveillance of bladder cancer: A phase III, comparative, multicenter study. J Urol 2018;199:1158-1165.

Original Publication Date: 2020 